\title{
Emergency Use Authorization for Remdesivir and Its Potential Implications
}

\author{
Cassius lyad Ochoa Chaar, MD, MS ${ }^{1}$ (1) $\cdot$ Robert Makuch, $\mathrm{PhD}^{2}$
}

Received: 25 June 2020 / Accepted: 24 August 2020 / Published online: 3 September 2020

(c) The Drug Information Association, Inc 2020

The COVID-19 pandemic is unraveling as the most devastating disease impacting humanity. The repercussions extend far beyond the sobering 15.3 million infections and 620,000 deaths worldwide, into a global economic free fall, exponential rise of unemployment, and disruption of social bonds [1]. At the center of this pandemic is a disease with no antidote or vaccine. The interim analysis of the NIH sponsored Adaptive COVID-19 Treatment Trial (ACTT) on April 27th, brought a spark of hope. The published results showed that treatment with remdesivir significantly decreased the time to recovery to 11 days compared to 15 days with placebo, a statistically significant reduction of $31 \%(P<0.001)$. Moreover, there was a trend towards decreased mortality at 14 days with the use of the drug ( $7.1 \%$ vs $11.9 \%)$ that did not reach statistical significance [2]. Unlike earlier reports from non-randomized studies [3], the ACTT was a prospectively randomized, controlled trial with adaptive design involving 1,063 patients and reviewed by an independent data and safety monitoring board (DSMB). Within 4 days, the compelling evidence lead the Food and Drug Administration (FDA) to grant Gilead Sciences Inc., the drug manufacturer, an Emergency Use Authorization (EUA). However, remdesivir remains a drug that is not approved in the USA as explicitly stated in the FDA letter [4]. In the absence of the EUA, patients would be limited to receiving remdesivir only in clinical trials or under a compassionate use agreement that requires special authorization from the FDA on an individual basis. This would be unnecessarily cumbersome with the current number of cases. The EUA enables physicians and hospitals to acquire the drug outside clinical trials especially

Cassius Iyad Ochoa Chaar

cassius.chaar@yale.edu

1 Division of Vascular Surgery, Department of Surgery, Yale University School of Medicine, 333 Cedar Street, Boardman 204, New Haven, CT 06510, USA

2 Director of Regulatory Affairs Track, Yale University School of Public Health, New Haven, CT, USA in the current circumstances of rapid disease propagation and lack of alternative effective therapy.

The number of clinical trials related to COVID-19 has exploded. The number of studies posted on clinicaltrials. gov increased from 4 in January 2020 to 1833 on May 31st [5] (Fig. 1). Remdesivir therapy was incorporated in only 29 trials. Hydroxychloroquine is the most commonly studied medication in 207 trials. Direct inhibition of COVID-19 replication is being investigated with lopinavir/ritonavir (69 trials), a combination used to treat human immunodeficiency virus (HIV). Several therapies aim to interfere with the disproportionate inflammatory response triggered by the virus either by using steroids like methylprednisolone ( 21 trials) or targeting key mediators such as interleukin-6 (IL-6). Tocilizumab (46 trials) is an antibody to IL-6 while sarilumab (14 trials) inhibits its receptor. Other antimicrobials such as azithromycin (91 trials), ivermectin (18 trials), and doxycycline (5 trials) are being used to treat co-infections or directly inhibit the virus based on speculative mechanisms of action. Alternative strategies focus on boosting the antiviral immunity through direct modulators such as interferon (59 trials) or a vaccine (106 trials).

Principal investigators, pharmaceutical companies, and regulatory bodies around the globe will face major ethical dilemmas. In 345 trials, patients with COVID-19 may be randomized to a placebo arm. A total of 1804 studies are enrolling patients to receive various therapies without formal inclusion of remdesivir based on the initial clinical designs. The EUA makes remdesivir legally available for patients and physicians and provides the opportunity for other trials to adapt their treatment protocol to incorporate it. However, it is uncertain in the immediate future whether remdesivir production can meet the rapidly growing demand. While the company expected producing 140,000 rounds of its 10-day treatment course by July, the number of active infections have reached several millions worldwide $[1,6]$. Remdesivir is administered intravenously in a hospital setting and is currently approved only for a subgroup of patients with 


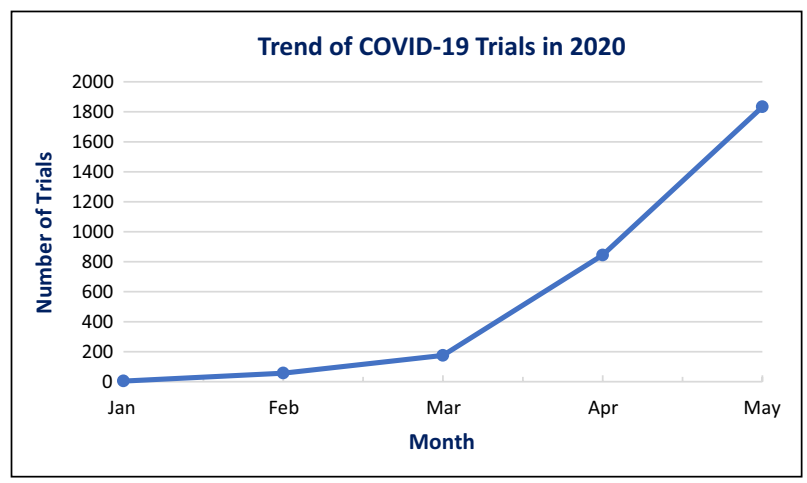

Figure 1. Number of Clinical Trials Related to COVID-19 Posted on clinicaltrials.gov.

severe disease requiring oxygen supplementation. Patient selection and allocation of the drug could be challenging at times of scarcity.

Early stages of drug development during the AIDS epidemic also involved the testing of a large number of competing agents with the early acceptance of just one NIH-supported compound. The approval of the first drug azidothymidine (AZT) for the treatment of HIV came after early halting of a phase 2 prospectively randomized, controlled clinical trial comparing it to placebo in September 1986. Back then, FDA approval of the drug was expedited and required 6 months for finalization until March 1987. It was based on one clinical trial demonstrating significantly increased survival and decrease in opportunistic infections compared to placebo [7]. Despite significant known toxicity and incomplete data on exact dosages, the FDA took an appropriate step to provide treatment for HIV infected patients with no other options for a lethal disease [8]. As a member serving on the DSMB (RM) of the original AZT vs. placebo study, the committee struggled with the issue of stopping the study early because its termination would impact the design of all future studies in terms of control group selection. With the usual Type 1 error rate selection of 0.05 , the concern was whether this one trial was a false positive. If so, an ineffective treatment would become the standard of care for treating HIV. Similar concerns remain with remdesivir in the absence of a consistent survival advantage and the publication of randomized data showing no signal of benefit [2,9]. Also, the ACTT was criticized for delay in initiation of therapy as well as the absence of information regarding concomitant medications to treat COVID-19. However, additional data from large observational studies are starting to emerge showing a significant decrease in mortality with remdesivir therapy (OR 0.38 [0.22-0.68]) after adjusting for patient comorbidities and concomitant treatments [10].
As with development of therapeutic options for different stages of HIV infection, the determination of optimal therapy for specific groups of COVID-19 patients will require time and must be guided by science and patience. The initial FDA support of hydroxychloroquine, followed later by its recommendation that hydroxychloroquine was not effective against COVID-19, served as a recurring reminder that regulatory decisions cannot be used to tame public emotions, media overstatement, or political agendas. Our compass in this challenging period remains the Hippocratic oath to put patients' health first based on the best available scientific knowledge at the time, recognizing that the standard of care is a rapidly moving target that we need to adapt to on a regular basis. The EUA for remdesivir is just the beginning of a long roller-coaster ride and a dim light in an abyss of dark statistics.

\section{References}

1. World Health Organization. Coronavirus disease 2019 (COVID19)_situation report. https://www.who.int/emergencies/diseases/ novel-coronavirus-2019/situation-reports. Accessed 30 May 2020.

2. Beigel JH, Tomashek KM, Dodd LE, et al. Remdesivir for the treatment of covid-19-preliminary report. N Engl J Med. 2020. https://doi.org/10.1056/NEJMoa2007764.

3. Gautret P, Lagier JC, Parola P, et al. Hydroxychloroquine and azithromycin as a treatment of COVID-19: results of an openlabel non-randomized clinical trial. Int J Antimicrob Agents. 2020;2020:105949.

4. Food and Drug Administration. Emergency use authorization for remdesivir. https://www.fda.gov/media/137564/download. Accessed 30 May 2020.

5. National Institute of Health USNLoM. clinicaltrials.gov. https:// clinicaltrials.gov/ct2/results?cond=COVID-19. Accessed 31 May 2020.

6. Statnews MH. Gilead CEO: we're going to make sure that access is not an issue with remdesivir. https://www.statnews. com/2020/04/29/gilead-ceo-were-going-to-make-sure-that-acces s-is-not-an-issue-with-remdesivir/. Accessed 30 May 2020.

7. Fischl MA, Richman DD, Grieco MH, et al. The efficacy of azidothymidine (AZT) in the treatment of patients with AIDS and AIDS-related complex A double-blind, placebo-controlled trial. N Engl J Med. 1987;317:185-91.

8. Brook I. Approval of zidovudine (AZT) for acquired immunodeficiency syndrome. A challenge to the medical and pharmaceutical communities. JAMA 1987;258:1517.

9. Wang Y, Zhang D, Du G, et al. Remdesivir in adults with severe COVID-19: a randomised, double-blind, placebo-controlled, multicentre trial. Lancet. 2020;395:1569-78.

10. Olender S, Perez KK, Go AS, Balani B, et al. Remdesivir for severe COVID-19 versus a cohort receiving standard of care. Clin Infect Dis. 2020. https://doi.org/10.1093/cid/ciaa1041. 